\title{
Observation of changes in left atrial and left ventricular function in patients with hypertensive disorders of pregnancy based on myocardial strain
}

\author{
Xinping Liu, Kaicheng Duan, Fengqin Zhu, Hui Zhang, Shu Zhang \\ Department of Ultrasound, The Fuyang Affiliated Hospital of Anhui Medical University, Fuyang, China \\ Contributions: (I) Conception and design: X Liu; (II) Administrative support: X Liu; (III) Provision of study materials or patients: X Liu, K Duan, F \\ Zhu; (IV) Collection and assembly of data: H Zhang, S Zhang; (V) Data analysis and interpretation: X Liu; (VI) Manuscript writing: All authors; (VII) \\ Final approval of manuscript: All authors. \\ Correspondence to: Xinping Liu. Department of Ultrasound, The Fuyang Affiliated Hospital of Anhui Medical University, Fuyang 236000, China. \\ Email: 39539686@qq.com.
}

Background: To observe the changes of left atrial and left ventricular function in patients with hypertensive disorders of pregnancy (HDP) based on myocardial strain.

Methods: A total of 66 HDP patients admitted to our hospital were retrospectively analyzed, and 36 normal pregnant admitted during the same period women were selected as the control group. The maximum volume of the left atrium $\left(\mathrm{LAV}_{\text {max }}\right)$, minimum volume of the left atrium $\left(\mathrm{LAV}_{\min }\right)$, left atrial active ejection fraction (LAAEF), mitral ratio of peak early to late diastolic filling velocity (E/A), and left ventricular active ejection fraction (LVEF) were measured by conventional echocardiography. The peak systolic strain rate (SRs) of each wall of the left atrium during early systole (SRe) and late diastole (SRa) was detected by speckletracking imaging (STI). The longitudinal (LS), radial (RS), and circumferential strain (CS) parameters of each wall of the left ventricle were also measured. The above parameters were compared between the two groups, and the correlation between mean SRa (mSRa) and LAAEF as well as left ventricular global longitudinal strain (GLS) and LVEF in HDP patients was analyzed.

Results: $\mathrm{LAV}_{\max }, \mathrm{LAV}_{\min }$, and LAAEF in the HDP group were higher than those in the control group, while the E/A ratio was lower than that in control group (all $\mathrm{P}<0.05$ ). However, there was no significant difference in LVEF between the two groups ( $\mathrm{P}>0.05)$. In the HDP group, the absolute values of SRs and SRe in each wall of the left atrium were lower than those in the control group, while the absolute values of SRa were higher than those in the control group. In addition, the absolute values of LS, CS, and RS values in each wall of left ventricle in the HDP group were lower than those in the control group (all $\mathrm{P}<0.05)$. Pearson correlation analysis showed that mSRa was negatively correlated with LAAEF $(r=-0.895, P=0.000)$ and that left ventricular long-axis GLS was negatively correlated with LVEF ( $\mathrm{r}=-0.646, \mathrm{P}=0.000)$ in HDP patients.

Conclusions: According to the STI results, HDP patients experience significant left atrial and left ventricular myocardial strain injury. Therefore, monitoring of cardiac function and early intervention should be strengthened in clinical practice.

Keywords: Hypertensive disorders of pregnancy (HDP); speckle-tracking imaging (STI); left atrium; left ventricle

Submitted Dec 03, 2020. Accepted for publication Jan 22, 2021.

doi: 10.21037/apm-20-2573

View this article at: http://dx.doi.org/10.21037/apm-20-2573 


\section{Introduction}

Hypertensive disorders of pregnancy (HDP) is a major risk factor for perinatal complications and death in pregnant women. This disease is generally manifested as hypertension and proteinuria after 20 weeks of gestation, or accompanied by dysfunction of other terminal organs and even coma and convulsions (1). Epidemiological studies have found that the incidence of HDP is about 7.0-12.0\%, which is related to the age of pregnant women, and whether they are primiparous or multiparous (2). In China, the natimortality rate of pregnant women with HDP is significantly higher than that reported in developed countries, which may be due to the differences in healthcare guidelines and prenatal care (3). Cardiac dysfunction is common in HDP patients, and studies have confirmed that cardiac structure and function changes may occur in the early stage of the disease (4). Therefore, monitoring cardiac function is crucial for disease evaluation. Echocardiography is a noninvasive test of cardiac function, which is economical, realtime, and radiation-free. However, conventional ultrasound can only reflect the inner diameter changes of the ventricle and atrium, but cannot accurately assess regional myocardial function. Speckle-tracking imaging (STI) is a technique that tracks the scattered speckles in a specific region, thus calculating their motion trajectory. STI functions independently of the angle of beam and myocardial motion, conferring it with a unique advantage over other ultrasound methods. In previous studies, STI has been mainly applied to assess cardiac function in hypertension and coronary heart disease, but it has rarely applied to evaluate left ventricular myocardial function in HDP patients. In this study, STI was therefore used to investigate changes of left atrial and left ventricular myocardial strain, so as to provide a reference for clinical diagnosis and treatment.

We present the following article in accordance with the MDAR checklist (available at http://dx.doi.org/10.21037/ apm-20-2573).

\section{Methods}

\section{Patients and grouping}

A total of 66 HDP patients admitted to our hospital between December 2017 and March 2020 were retrospectively selected. They were aged 19-48 years (average: $30.26 \pm 6.64$ years), with 27-42 weeks of gestation (average: $35.89 \pm 3.78$ weeks) and a BMI of $15.79-39.60 \mathrm{~kg} / \mathrm{m}^{2}$ (average: $27.39 \pm 4.90 \mathrm{~kg} / \mathrm{m}^{2}$ ). In accordance with the guidelines for the diagnosis and treatment of hypertension in pregnancy (2015) (5), all subjects were diagnosed with HDP base on their medical history, symptoms, signs, and related auxiliary examinations. Among the patients, 18 had gestational hypertension, 22 had mild preeclampsia, and 26 had severe preeclampsia. Meanwhile, 36 normal pregnant women who were admitted during the same period as HDP patients were selected as the control group. They were aged 18-42 years (average: $28.42 \pm 5.45$ years), with $29-42$ weeks of gestation (average: $34.47 \pm 3.24$ weeks) and a BMI of 20.18-36.69 kg/m² (average: $28.83 \pm 4.32 \mathrm{~kg} / \mathrm{m}^{2}$ ).

The inclusion criteria were as follows: (I) singleton pregnancy, (II) conventional echocardiography and STI, and (III) age $\geq 18$ years. The exclusion criteria were as follows: (I) thorax deformity, (II) chronic hypertension, (III) gestational diabetes, (IV) liver and kidney dysfunction due to other causes, (V) organic or functional heart disease, (VI) severe arrhythmia, (VII) severe anemia, and (VIII) fetal structural or chromosomal abnormalities.

There were no significant differences in age $(t=-1.421$, $\mathrm{P}=0.158)$, gestational age $(\mathrm{t}=1.993, \mathrm{P}=0.049)$, or $\mathrm{BMI}$ $(\mathrm{t}=1.477, \mathrm{P}=0.143)$ between the two groups $(\mathrm{P}>0.05)$. The study was approved by the ethics committee of our hospital (No. KY2020008). All procedures performed in this study involving human participants were in accordance with the Declaration of Helsinki (as revised in 2013). Individual consent for this retrospective analysis was waived.

\section{Ultrasound and image analysis}

A GE Vivid E9 ultrasound machine (GE Healthcare, USA) was used, equipped with an M5S two-dimensional phased array cardiac probe, with a frequency of $1.7-$ 3.3 $\mathrm{MHz}$ and a frame rate of $60-80$ frames/s. Three-channel electrocardiographic leads were connected. EchoPAC software was used for offline analysis of the data. The subjects were asked to lie in the left lateral decubitus position, and the probe was placed on the left sternal border and apical region. According to the criteria introduced by the American Society of Echocardiography (ASE), conventional echocardiography was adopted to measure and calculate the maximum volume of the left atrium $\left(\mathrm{LAV}_{\max }\right)$, minimum volume of the left atrium $\left(\mathrm{LAV}_{\min }\right)$, left atrial active ejection fraction (LAAEF), mitral ratio of peak early to late diastolic filling velocity (E/A), and left ventricular active ejection fraction (LVEF). Dynamic images of three cardiac cycles from apical four-chamber, left ventricular two-chamber, and left ventricular three-chamber views were recorded, respectively. In addition, left ventricular 
Table 1 Comparison of conventional echocardiography parameters between the two groups

\begin{tabular}{|c|c|c|c|c|c|c|}
\hline Group & Case & $\mathrm{LAV}_{\text {max }}(\mathrm{mL})$ & $\mathrm{LAV}_{\text {min }}(\mathrm{mL})$ & LAAEF (\%) & $E / A$ & LVEF (\%) \\
\hline HDP group & 66 & $44.97 \pm 4.25$ & $16.36 \pm 4.02$ & $39.15 \pm 4.36$ & $1.03 \pm 0.29$ & $60.44 \pm 3.17$ \\
\hline$t$ & & -12.384 & -5.818 & -4.561 & 2.721 & -0.670 \\
\hline$P$ & & 0.000 & 0.000 & 0.000 & 0.008 & 0.504 \\
\hline
\end{tabular}

$\mathrm{LAV}_{\text {max }}$, maximum volume of the left atrium; $\mathrm{LAV}_{\text {min }}$, minimum volume of the left atrium; LAAEF, left atrial active ejection fraction; $E / A$, mitral ratio of peak early to late diastolic filling velocity; LVEF, left ventricular active ejection fraction; HDP, hypertensive disorders of pregnancy.

Table 2 Comparison of SRs of each left atrial wall between the two groups $\left(\mathrm{s}^{-1}, \bar{x} \pm \mathrm{s}\right)$

\begin{tabular}{lcccccc}
\hline Group & Case & Atrial septum & Lateral wall & Inferior wall & Anterior wall & Posterior wall \\
\hline Control group & 36 & $2.46 \pm 0.63$ & $2.38 \pm 0.58$ & $2.39 \pm 0.93$ & $2.23 \pm 0.80$ & $2.17 \pm 0.72$ \\
HDP group & 66 & $2.03 \pm 0.88$ & $1.73 \pm 0.53$ & $1.98 \pm 0.37$ & $1.61 \pm 0.65$ & $1.78 \pm 0.55$ \\
$t$ & & 2.823 & 5.779 & 2.550 & 4.261 & 3.009 \\
$P$ & 0.006 & 0.000 & 0.015 & 0.000 & 0.003 \\
\hline
\end{tabular}

SRs, strain rate of systolic; HDP, hypertensive disorders of pregnancy.

regional systolic longitudinal strain (LS), radial strain (RS), circumferential strain (CS), and left ventricular global systolic longitudinal strain (GLS) were measured. The left atrial endocardium was traced, so as to obtain the peak longitudinal strain rate of systole (SRs), the peak longitudinal strain rate of early diastole (SRe), and the peak longitudinal strain rate of late diastole (SRa) of each left atrial wall.

\section{Statistical analysis}

SPSS 22.0 software (IBM Corp., Armonk, NY, USA) was used for statistical analysis. All measurement data are presented as $\bar{x} \pm$ s. Independent samples t-test was used to compare measurement data between the two groups. Pearson analysis was used to analyze the correlation between mean SRa (mSRa) and LAAEF in each wall of left atrium, and the correlation between left ventricular longaxis GLS and LVEF in HDP patients. A $P$ value $<0.05$ was considered to be statistically significant.

\section{Results}

\section{Comparison of conventional echocardiography parameters between the two groups}

$\mathrm{LAV}_{\max }, \mathrm{LAV}_{\text {min }}$, and LAAEF were higher while E/A was lower in the HDP group compared with the control group $(\mathrm{P}<0.05)$. However, there was no significant difference in LVEF between the two groups $(\mathrm{P}>0.05)$ (Table 1).

\section{Comparison of STI results of left atrium between the two groups}

The absolute values of SRs and SRe of each left atrial wall in the HDP group were lower than those of the control group, while the absolute values of SRa were higher than those of the control group $(\mathrm{P}<0.05)$ (Tables 2-4).

\section{Comparison of STI results of the left ventricle between the two groups}

The absolute values of LS and CS, and RS values in each ventricular wall of the HDP group were lower than those of the control group, and the differences were statistically significant $(\mathrm{P}<0.05)$ (Tables 5-7, Figures 1-4).

\section{Correlation between mSRa and LAAEF in HDP patients}

The Pearson correlation analysis showed that $\mathrm{mSRa}$ was negatively correlated with LAAEF in HDP patients $(\mathrm{r}=-0.895, \mathrm{P}=0.000)$ (Figure 5). 
Table 3 Comparison of SRe of each left atrial wall between the two groups $\left(\mathrm{s}^{-1}, \bar{x} \pm \mathrm{s}\right)$

\begin{tabular}{lcccccc}
\hline Group & Case & Atrial septum & Lateral wall & Inferior wall & Anterior wall & Posterior wall \\
\hline Control group & 36 & $-2.20 \pm 0.57$ & $-2.02 \pm 0.65$ & $-2.03 \pm 0.65$ & $-2.18 \pm 0.51$ & $-2.25 \pm 0.79$ \\
HDP group & 66 & $-1.54 \pm 0.89$ & $-1.69 \pm 0.44$ & $-1.41 \pm 0.39$ & $-1.53 \pm 0.31$ & $-1.82 \pm 0.76$ \\
$t$ & & -4.568 & -2.696 & -5.265 & -6.990 & -2.728 \\
P & 0.000 & 0.009 & 0.000 & 0.000 & 0.008 \\
\hline
\end{tabular}

$\mathrm{SRe}$, strain rate of early systole; HDP, hypertensive disorders of pregnancy.

Table 4 Comparison of SRa of each left atrial wall between the two groups $\left(\mathrm{s}^{-1}, \bar{x} \pm \mathrm{s}\right)$

\begin{tabular}{|c|c|c|c|c|c|c|}
\hline Group & Case & Atrial septum & Lateral wall & Inferior wall & Anterior wall & Posterior wall \\
\hline HDP group & 66 & $-2.21 \pm 0.55$ & $-2.31 \pm 0.90$ & $-1.97 \pm 0.39$ & $-1.61 \pm 0.37$ & $-1.83 \pm 0.73$ \\
\hline$t$ & & 4.731 & 2.173 & 7.249 & 4.431 & 4.649 \\
\hline$P$ & & 0.000 & 0.032 & 0.000 & 0.000 & 0.000 \\
\hline
\end{tabular}

$\mathrm{SRa}$, strain rate of late diastole; HDP, hypertensive disorders of pregnancy.

Table 5 Comparison of the peak systolic LS of the left ventricle between the two groups $(\%, \bar{x} \pm s)$

\begin{tabular}{lccccccc}
\hline Group & Case & Anterior septum & Posterior septum & Lateral wall & Inferior wall & Anterior wall & Posterior wall \\
\hline Control group & 36 & $-23.58 \pm 3.28$ & $-24.14 \pm 3.66$ & $-23.60 \pm 4.91$ & $-24.01 \pm 4.82$ & $-22.46 \pm 5.37$ & $-23.46 \pm 4.99$ \\
HDP group & 66 & $-15.43 \pm 3.25$ & $-15.13 \pm 2.69$ & $-15.35 \pm 4.02$ & $-16.10 \pm 4.22$ & $-16.44 \pm 4.58$ & $-15.92 \pm 4.27$ \\
$t$ & & -12.068 & -12.985 & -9.141 & -8.595 & -5.959 & -8.031 \\
P & 0.000 & 0.000 & 0.000 & 0.000 & 0.000 & 0.000 \\
\hline
\end{tabular}

LS, longitudinal strain; HDP, hypertensive disorders of pregnancy.

Table 6 Comparison of the peak systolic RS of the left ventricle between the two groups (\%, $\bar{x} \pm \mathrm{s}$ )

\begin{tabular}{lccccccc}
\hline Group & Case & Anterior septum & Posterior septum & Lateral wall & Inferior wall & Anterior wall & Posterior wall \\
\hline Control group & 36 & $23.70 \pm 5.51$ & $24.37 \pm 5.43$ & $24.44 \pm 4.84$ & $22.97 \pm 6.04$ & $24.27 \pm 4.63$ & $24.00 \pm 4.77$ \\
HDP group & 66 & $15.61 \pm 4.61$ & $15.09 \pm 5.66$ & $16.12 \pm 4.62$ & $15.44 \pm 3.82$ & $15.87 \pm 3.54$ & $16.59 \pm 3.68$ \\
$t$ & & 7.893 & 8.022 & 8.555 & 6.778 & 10.239 & 8.736 \\
P & 0.000 & 0.000 & 0.000 & 0.000 & 0.000 & 0.000 \\
\hline
\end{tabular}

RS, radial strain; HDP, hypertensive disorders of pregnancy.

Table 7 Comparison of the peak systolic CS of left ventricle between the two groups $(\%, \bar{x} \pm s)$

\begin{tabular}{lccccccc}
\hline Group & Case & Anterior septum & Posterior septum & Lateral wall & Interior wall & Anterior wall & Posterior wall \\
\hline Control group & 36 & $-23.21 \pm 4.70$ & $-24.26 \pm 4.74$ & $-24.97 \pm 4.46$ & $-25.62 \pm 4.92$ & $-23.20 \pm 5.47$ & $-22.64 \pm 5.16$ \\
HDP group & 66 & $-17.05 \pm 3.43$ & $-17.08 \pm 3.92$ & $-16.01 \pm 4.05$ & $-16.41 \pm 4.19$ & $-15.43 \pm 4.07$ & $-15.89 \pm 4.06$ \\
$t$ & & -7.590 & -8.205 & -10.311 & -9.967 & -7.473 & -7.268 \\
$P$ & 0.000 & 0.000 & 0.000 & 0.000 & 0.000 & 0.000 \\
\hline
\end{tabular}

CS, circumferential strain; HDP, hypertensive disorders of pregnancy. 


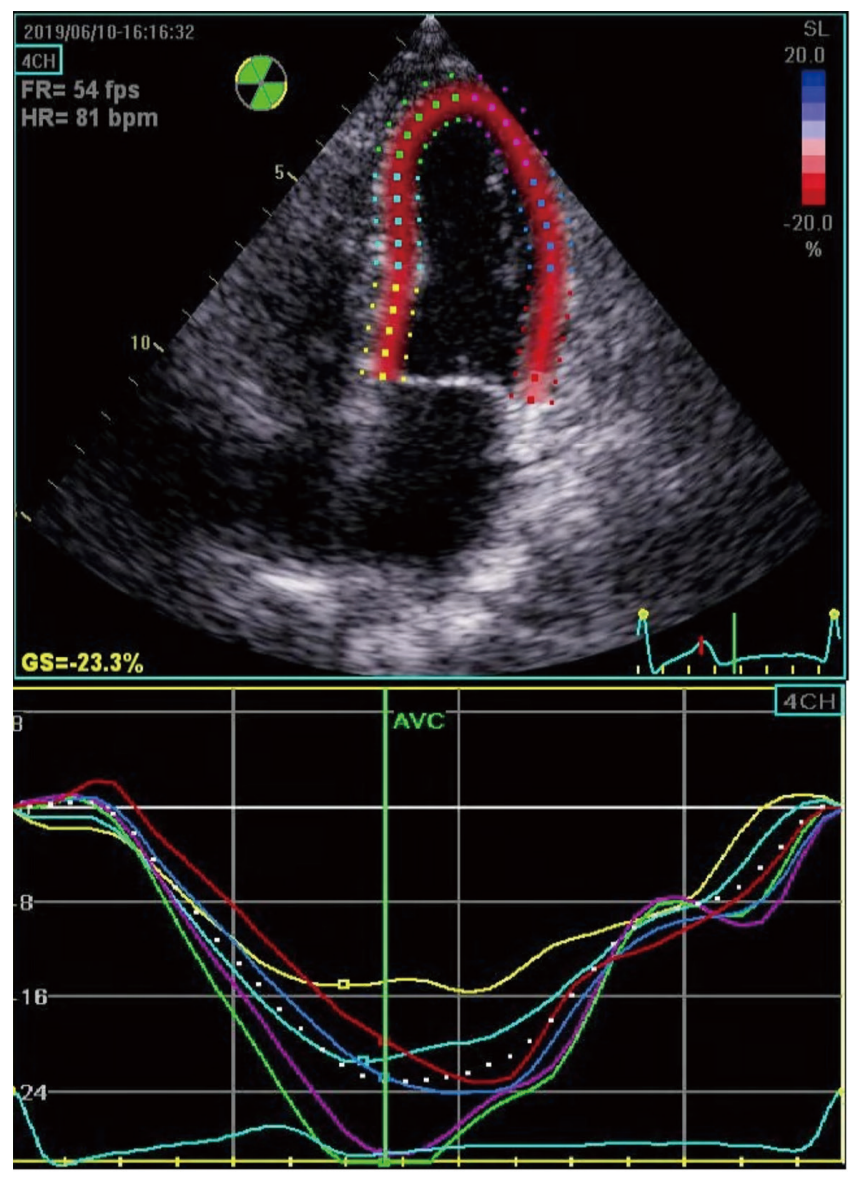

Figure 1 Longitudinal strain curve of the left ventricular myocardium in normal pregnant women.

\section{Correlation between GLS and LVEF in HDP patients}

The Pearson correlation analysis showed that there was a negative relationship between GLS and LVEF in HDP patients ( $\mathrm{r}=-0.646, \mathrm{P}=0.000)$ (Figure 6).

\section{Discussion}

\section{Conventional echocardiography parameters}

Left atrial volume has been proven to be an influencing factor of cardiac output and an effective prognostic indicator of a variety of cardiovascular diseases (6). In this study, $L A V_{\text {max }}$ and $L A V_{\text {min }}$ were significantly increased in the HDP group compared with the control group, indicating that the left atrium was remodeled and the volume was enlarged. It is possible that hypertension leads to increased afterload, resulting in obstruction and retention of left atrial blood discharge. Meanwhile, due to the impairment

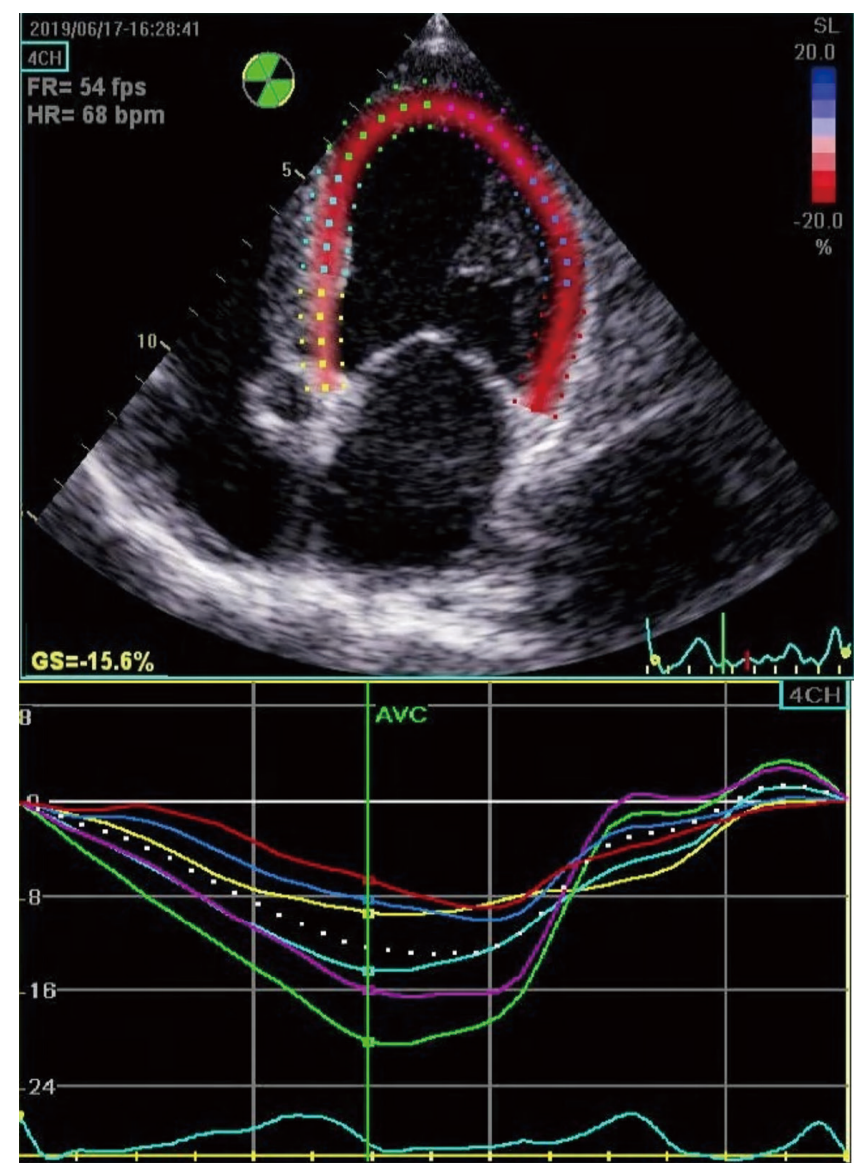

Figure 2 Longitudinal strain curve of the left ventricular myocardium in HDP patients. HDP, hypertensive disorders of pregnancy.

of left ventricular diastolic function, the left atrial pressure increases compensatorily to ensure left ventricular filling. The left atrial wall is thus weakened, and eventually, the left atrium becomes enlarged. Studies have shown that in a certain range, the decrease of $\mathrm{E} / \mathrm{A}$ ratio is related to the degree of impaired left ventricular diastolic function (7). In this study, E/A ratios were lower in the HDP group than those in the control group. The decreased ratio suggests that diastolic function is weakened, which may be related to an increased volume load and decreased ventricular compliance. In addition, we found no significant difference in LVEF between the HDP group and control group. Butnariu et al. (8) observed that abnormality of left ventricular systolic function occurred later than that of left ventricular diastolic function; therefore, when the left ventricular diastolic function was impaired, the systolic function was still at a normal level. In the study of Mostafavi 


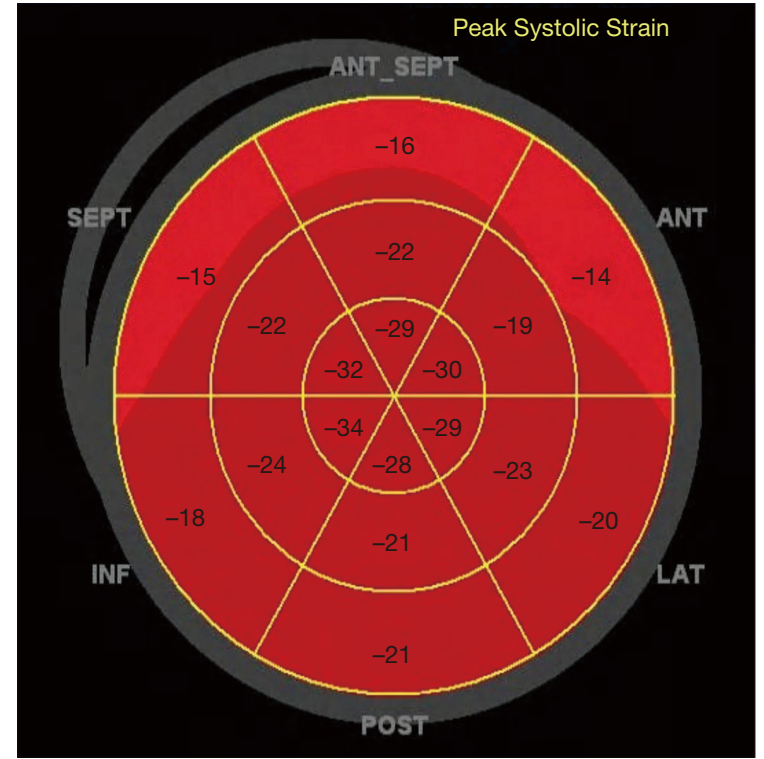

Figure 3 Bull's eye diagram of the longitudinal strain of the left ventricular myocardium in normal pregnant women.

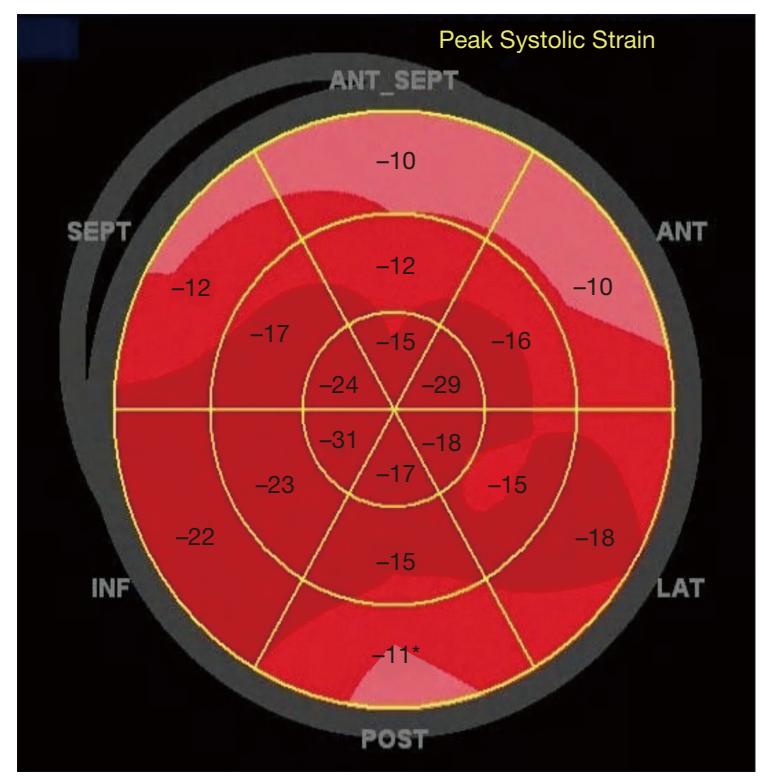

Figure 4 Bull's eye diagram of the longitudinal strain of the left ventricular myocardium in HDP patients. HDP, hypertensive disorders of pregnancy.

et al. (9), the left ventricular systolic function in normal pregnant women was also impaired to varying degrees, and thus LVEF may not be sufficiently sensitive to evaluate the left ventricular systolic function in HDP patients. In terms of LAAEF, we found that it was higher in the HDP group than that in the normal group. Global left atrial systolic function was positively correlated with volume before active ejection; indeed, the increase of LAV and LAAEF has been demonstrated to be an important supplement to left ventricular filling in HDP patients with left ventricular diastolic dysfunction (10).

\section{Two-dimensional STI of left atrium}

Atrial function includes the following three functions: reservoir function involves the passive filling during atrial diastole; conduit function involves the blood flow from the pulmonary veins into the left ventricle through the left atrium during the early phase of ventricular diastole; booster pump function involves the active contraction of the left atrium during the late phase of ventricular diastole. SRs, SRe, and SRa correspond to reservoir, conduit, and booster pump functions, respectively. The SRs in this study was decreased in HDP patients, suggesting a decline in left atrial reservoir function. Other studies have shown that longterm hypertension and increased left ventricular afterload in HDP patients lead to an increase in left atrial residual blood volume and excessive synthesis of myocardial interstitial collagen fibers in the atrial wall, resulting in a decrease in atrial wall extension and compliance during diastole (11). Furthermore, the absolute value of SRe in our study was decreased in HDP patients, indicating a decrease in left atrial conduit function. In HDP patients, the blood pressure was increased and left ventricular diastolic function was decreased, which affected the pressure differences between the left atrium and left ventricle and between the pulmonary vein and left ventricle, thus resulting in a decrease in the blood volume flowing from the pulmonary veins to the left ventricle in the early ventricular diastole. Additionally, the absolute value of SRa in our study was increased in HDP patients, suggesting an enhancement in left atrial booster pump function, which may be an early compensatory manifestation for ventricular underfilling. Similar to our study, Yang et al. (12) also found that the absolute values of SRs and SRe were decreased while the absolute values of SRa were increased in hypertensive patients with left ventricular hypertrophy, indicating that the regularity of left atrial systolic and diastolic motion was weakened and the booster pump function was compensatorily enhanced.

\section{Two-dimensional STI of left ventricle}

Our results showed that the absolute value of LS was 


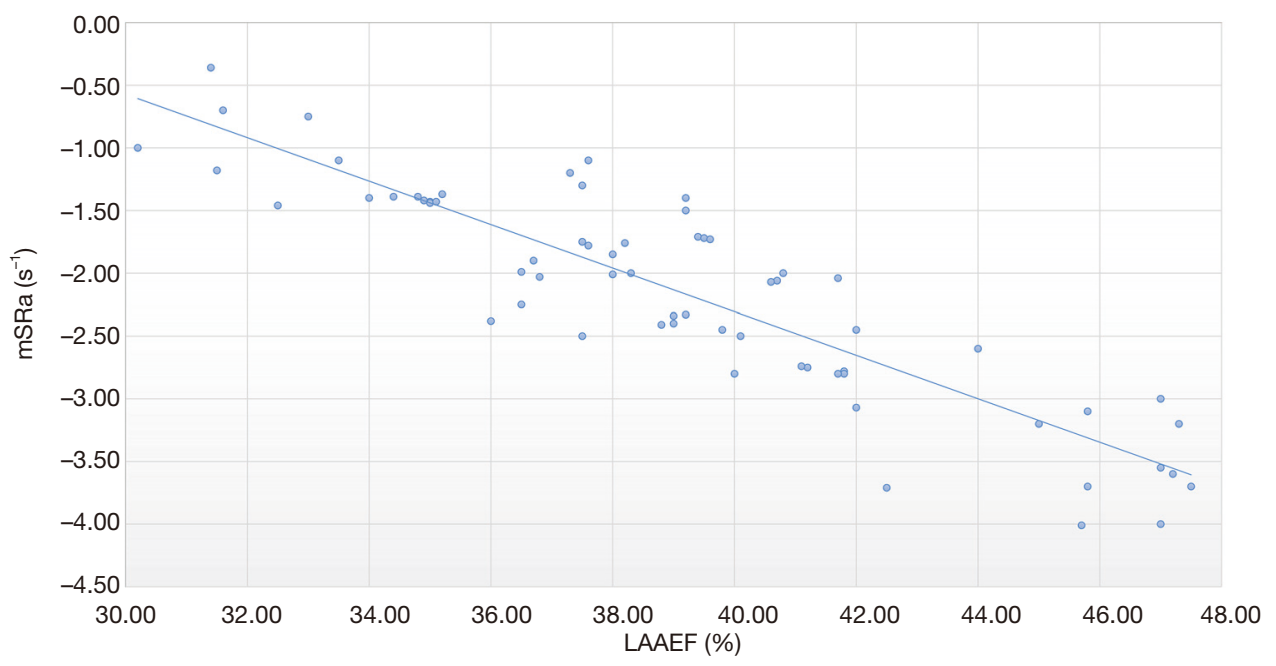

Figure 5 Correlation between mSRa and LAAEF in HDP patients. mSRa, mean SRa; LAAEF, left atrial active ejection fraction; HDP, hypertensive disorders of pregnancy.

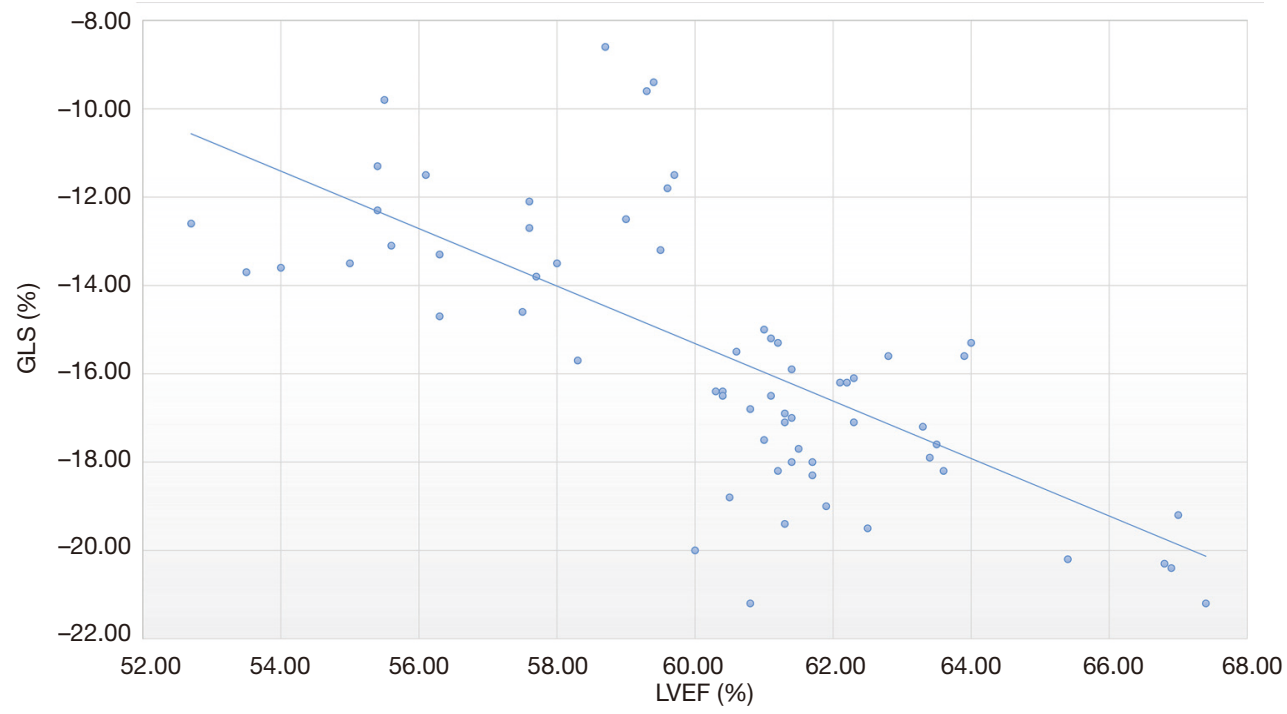

Figure 6 Correlation between GLS and LVEF in HDP patients. GLS, global longitudinal strain; LVEF, left ventricular active ejection fraction; HDP, hypertensive disorders of pregnancy.

decreased in the HDP group compared with the control group, suggesting a decrease in regional systolic function. Vascular endothelial injury is a characteristic pathological change of HDP, causing thrombosis and vasospasm, which in turn leads to myocardial ischemia and fibrosis (13). The oxygen consumption of the subendocardial myocardium is higher than that of the subepicardial myocardium, and the blood supply is mainly derived from ventricular diastolic function. However, the supply is insufficient in HDP patients due to the accelerated heart rate and shortened diastole. Studies have shown that longitudinal myocardial fibers are mainly distributed in the subendocardium, and contraction from the middle layer ventricular wall to the epicardial myocardium determines the radial strain of the myocardium, while the middle layer of myocardium is closely related to circumferential strain (14). In HDP patients, myocardial ischemia generally starts from the inner myocardium and gradually involves the outer myocardium. 
Therefore, after myocardial ischemia and fibrosis reach a certain threshold, myocardial injury in the middle and epicardial layers occurs, ultimately manifesting as decreased RS value and CS absolute value. Our study showed that absolute values of LS and CS, along with RS values, were decreased in the HDP group compared with the control group, which is consistent with the above study. In addition, the correlation analysis of this study showed that in HDP patients, mSRa was negatively correlated with LAAEF, while GLS was negatively correlated with LVEF, suggesting that mSRa and GLS are good indicators of global left atrial and ventricular systolic function, respectively, which is consistent with the results of Chen $e$ t al. (15).

\section{Summary and limitations}

The STI results of this study indicate that HDP patients experience significant left atrial and left ventricular myocardial strain injury. Therefore, cardiac function monitoring and early intervention should be strengthened in clinical practice. STI can detect cardiac function impairment before cardiac morphology changes, which is helpful for risk assessment and treatment guidance, thus reducing left heart failure and pulmonary edema, and improving pregnancy outcomes thereby. However, some limitations to the study should also be noted: (I) this was a single-center study with a limited number of samples. The clinical application value of STI technology in HDP patients requires further validation by multicenter and large-sample studies. (II) Only the overall HDP patients were selected as the subjects, and the differences in cardiac function among different types of HDP patients still need to be comprehensively assessed. (III) Two-dimensional STI is highly frame rate-dependent, and further analysis which includes combination with three-dimensional STI can improve the reliability of the study results.

\section{Acknowledgments}

Funding: None.

\section{Footnote}

Reporting Checklist: The authors have completed the MDAR reporting checklist. Available at http://dx.doi.org/10.21037/ apm-20-2573

Data Sharing Statement: Available at http://dx.doi. org/10.21037/apm-20-2573

Conflicts of Interest: All authors have completed the ICMJE uniform disclosure form (available at http://dx.doi. org/10.21037/apm-20-2573). The authors have no conflicts of interest to declare.

Ethical Statement: The authors are accountable for all aspects of the work in ensuring that questions related to the accuracy or integrity of any part of the work are appropriately investigated and resolved. All procedures performed in this study involving human participants were in accordance with the Declaration of Helsinki (as revised in 2013). This study was approved by the Ethics Committee of the Fuyang Affiliated Hospital of Anhui Medical University (No. KY2020008). Individual consent for this retrospective analysis was waived.

Open Access Statement: This is an Open Access article distributed in accordance with the Creative Commons Attribution-NonCommercial-NoDerivs 4.0 International License (CC BY-NC-ND 4.0), which permits the noncommercial replication and distribution of the article with the strict proviso that no changes or edits are made and the original work is properly cited (including links to both the formal publication through the relevant DOI and the license). See: https://creativecommons.org/licenses/by-nc-nd/4.0/.

\section{References}

1. Quitterer U, Fu X, Pohl A, et al. Beta-Arrestin1 Prevents Preeclampsia by Downregulation of Mechanosensitive AT1-B2 Receptor Heteromers. Cell 2019;176:318-33.e19.

2. Filipek A, Jurewicz E. Preeclampsia - a disease of pregnant women. Postepy Biochem 2018;64:232-9.

3. Ananth CV, Basso O. Impact of pregnancy-induced hypertension on stillbirth and neonatal mortality. Epidemiology 2010;21:118-23.

4. Orabona R, Sciatti E, Prefumo F, et al. Pre-eclampsia and heart failure: a close relationship. Ultrasound Obstet Gynecol 2018;52:297-301.

5. Pregnancy Hypertension Disease Group, Chinese Medical Association Obstetrics and Gynecology Branch. Guidelines for the diagnosis and treatment of hypertension in pregnancy (2015). Chinese Journal of Perinatal Medicine 2016;19:161-9.

6. Katsiki N, Mikhailidis DP, Papanas N. Left atrial volume: An independent predictor of cardiovascular outcomes. Int 
J Cardiol 2018;265:234-35.

7. Heiskanen JS, Ruohonen S, Rovio SP, et al. Determinants of left ventricular diastolic function-The Cardiovascular Risk in Young Finns Study. Echocardiography 2019;36:854-61.

8. Butnariu A, Zamfir C, Iancu M, et al. Systolic and Diastolic Function in Congestive Heart Failure Pediatric Patients. Rom J Intern Med 2016;54:37-46.

9. Mostafavi A, Tase Zar Y, Nikdoust F, et al. Comparison of left ventricular systolic function by $2 \mathrm{D}$ speckle-tracking echocardiography between normal pregnant women and pregnant women with preeclampsia. J Cardiovasc Thorac Res 2019;11:309-13.

10. Worley E, Rana B, Williams L, et al. Left ventricular diastolic dysfunction: identifying presence by left atrial function. Echo Res Pract 2018;5:97-104.

11. Tahir E, Starekova J, Muellerleile K, et al. Myocardial Fibrosis in Competitive Triathletes Detected by ContrastEnhanced CMR Correlates With Exercise-Induced Hypertension and Competition History. JACC Cardiovasc
Imaging 2018;11:1260-70.

12. Yang L, Qiu Q, Fang SH. Evaluation of left atrial function in hypertensive patients with and without left ventricular hypertrophy using velocity vector imaging. Int J Cardiovasc Imaging 2014;30:1465-71.

13. Zhao J, Peng W, Ran Y, et al. Dysregulated expression of ACTN4 contributes to endothelial cell injury via the activation of the p38-MAPK/p53 apoptosis pathway in preeclampsia. J Physiol Biochem 2019;75:475-87.

14. Sharif H, Ting S, Forsythe L, et al. Layer-specific systolic and diastolic strain in hypertensive patients with and without mild diastolic dysfunction. Echo Res Pract 2018;5:41-9.

15. Chen B, Guo W, Zhou LY, et al. Evaluation of left atrial function in patients with hypertrophic cardiomyopathy by real-time three-dimensional echocardiography and twodimensional speckle tracking imaging. Journal of Clinical Ultrasound in Medicine 2018;20:721-5.

(English Language Editor: J. Gray)
Cite this article as: Liu X, Duan K, Zhu F, Zhang H, Zhang S. Observation of changes in left atrial and left ventricular function in patients with hypertensive disorders of pregnancy based on myocardial strain. Ann Palliat Med 2021;10(1):606614. doi: 10.21037/apm-20-2573 